\title{
„Freundlichste Grüße aus der Augenklinik“ - Die alten Tübinger Augenkliniken auf Postkarten
}

\section{“Friendly Greetings from the Eye Clinic" - The Old Eye Hospitals in Tübingen on Postcards}

Vor allem Bauwerke zeugen vom Wirken der Menschheit. In Gebäuden spiegeln sich wandelnde technische Möglichkeiten, steigende Anforderungen an die Funktion und sich änderndes ästhetisches Empfinden wider. Dies gilt, vielleicht sogar in besonderer Weise, auch für (Augen-)Kliniken. Krankenhausbauten sind aber in erster Linie Orte der erhofften Besserung der Gesundheit oder gar der Heilung. Deshalb verwundert es nicht, dass sie in der Vergangenheit immer wieder als Motive für Ansichtskarten dienten. Aus einer Sammlung von 20 Ansichtskarten der ersten beiden Tübinger Universitäts-Augenkliniken sollen einige Beispiele präsentiert werden. Der Autor geht davon aus, dass die Sammlung die große Mehrzahl, aber nicht alle der jemals produzierten Ansichtskarten der Tübinger Augenkliniken umfasst.

Die erste Augenklinik befand sich in der Wilhelmstraße direkt gegenüber dem neuen Universitätshauptgebäude von 1846 [1-4]. Das Gebäude wurde primär nicht als Augenklinik errichtet, vielmehr 1872 vom ersten Tübinger Ordinarius, Albrecht Eduard Nagel (1833-1895), erworben und als Klinik eingerichtet. Im Jahre 1875 wurde diese vom Württembergischen Staat als Universitäts-Augenklinik mit 28 Betten übernommen. Trotz eines Anbaus 1884/85 blieb die Kapazität stets hinter dem Notwendigen zurück, die Klinik war deshalb nur bis 1908 in Betrieb. Das Gebäude ist erhalten und beherbergt heute u. a. die Universitätskasse. Die Ansichtskarte ( $>$ Abb. 1) zeigt die erste Universitäts-Augenklinik zusammen mit den damals existierenden, anderen Kliniken.

Die 2. Tübinger Augenklinik wurde auf einer Anhöhe hinter dem Universitätshauptgebäude errichtet und am 1. Januar 1909 mit 110 Betten in Betrieb genommen. Maßgeblicher Planer war Gustav von Schleich (1851-1928) [2-3,5-7], der das Ordinariat 1895 von Nagel übernommen

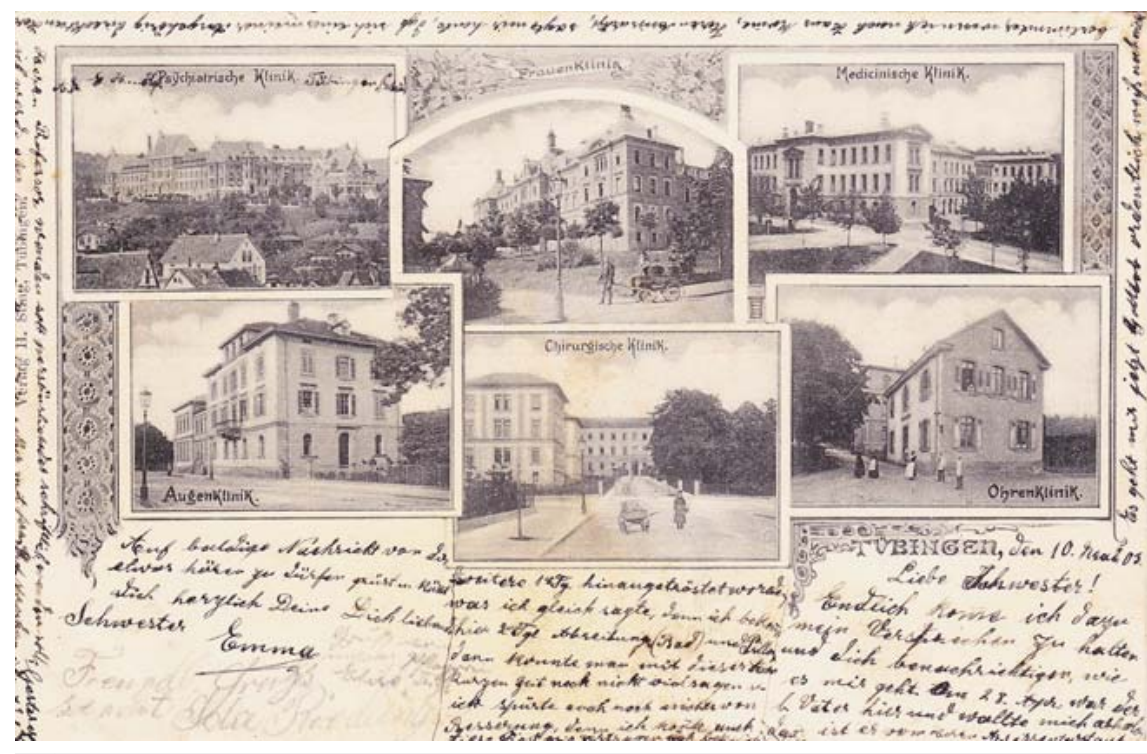

- Abb. 1 Erste Universitäts-Augenklinik Tübingen in der Wilhelmstraße (links unten). Da die Spezialisierung in der Medizin erst 40-50 Jahre zuvor begonnen hatte, gab es seinerzeit in Tübingen nur 6 Fachdisziplinen, die über ein eigenes Gebäude verfügten. Die Augenheilkunde als ältere Disziplin gehörte dazu. Datierung und Poststempel vom Mai 1903.

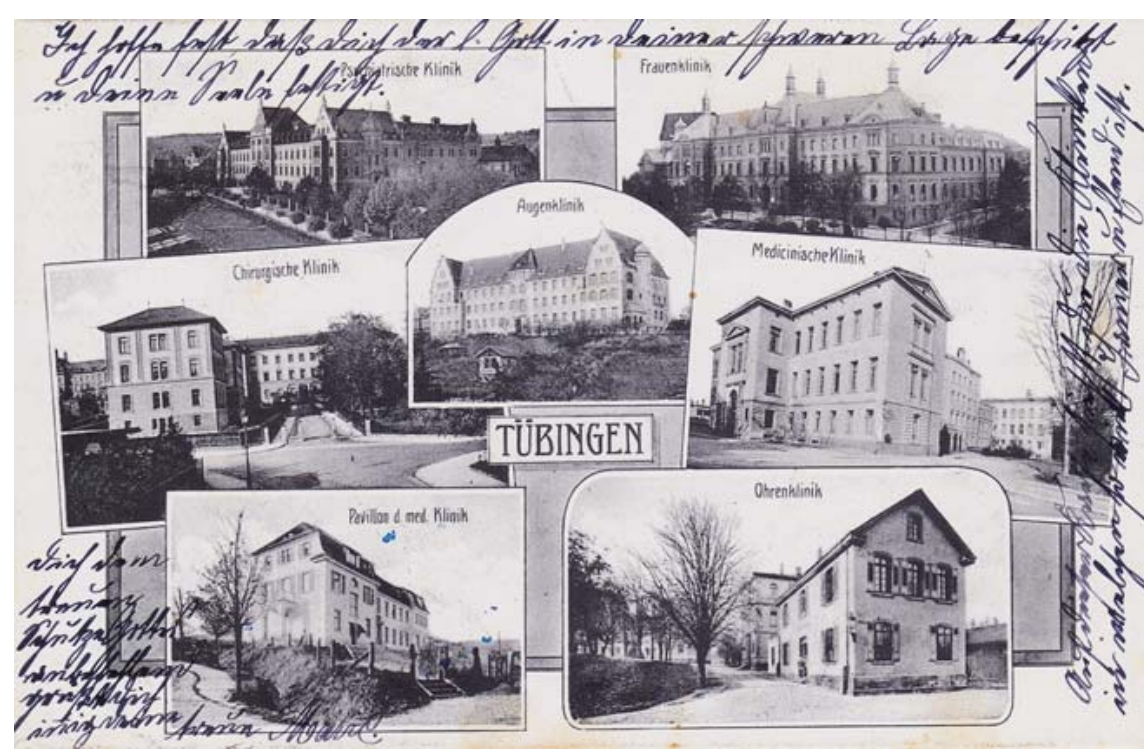

- Abb. 2 Wahrscheinlich, weil die neue 2. Augenklinik erst kurz zuvor in Betrieb genommen worden war, wurde sie im Zentrum dieser Postkarte mit allen Kliniken platziert. Datierung auf den Juli 1912. 


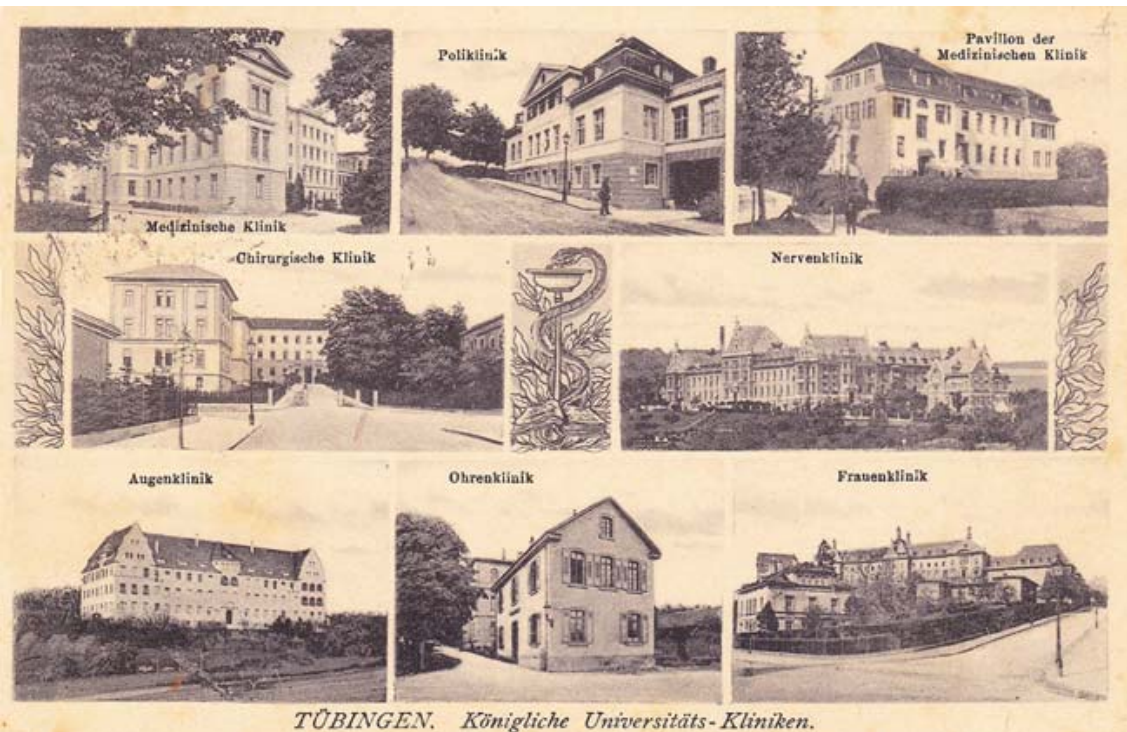

- Abb. 3 Tübinger Kliniken mit Augenklinik links unten. Der im Zentrum befindliche Äskulapstab verdeutlicht, dass die einzelnen Kliniken Teil eines Klinikums sind. Die Zeilen der Schreiberin wurden auf das Jahr 1920 datiert, jedoch stammt die Postkarte wegen des Aufdrucks „Königliche Universitäts-Kliniken“ aus der Zeit vor Ende 1918, denn seit der Abdankung König Wilhelms II. von Württemberg im November 1918 waren die Kliniken nicht mehr „königlich“.

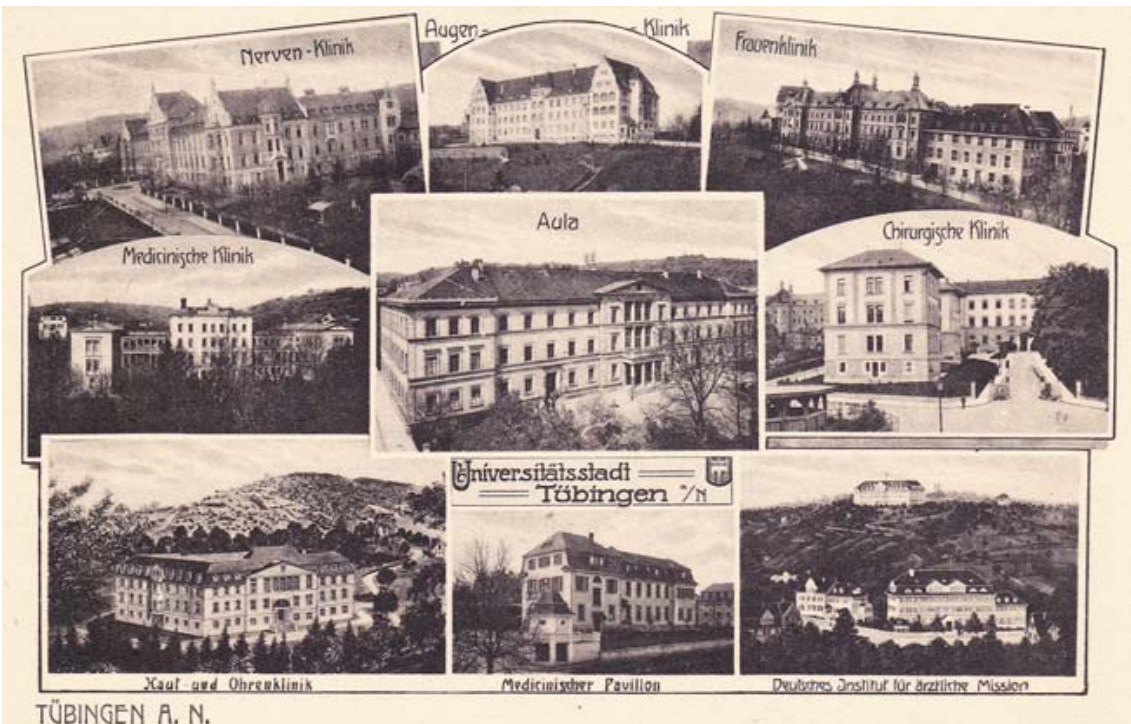

- Abb. 4 Tübinger Kliniken mit neuer Augenklinik oben mittig. Neu hinzugekommen ist die Hautklinik. Im Zentrum der Postkarte die Neue Aula als Sinnbild für die Universität, wodurch die Bedeutung der Kliniken als akademische Einrichtungen unterstrichen wird. Die Karte ist ungelaufen und deshalb ohne Datierung, dürfte aber aus den frühen 20er-Jahren stammen.

hatte und bis 1921 vertrat. Auf allen Ansichtskarten ist diese heute unter Denkmalschutz stehende Augenklinik mit ihrer repräsentativen, der Altstadt zugewandten Südseite fotografiert ( $\triangleright$ Abb. 211). Zum Teil erfolgte die Reproduktion wiederum gemeinsam mit anderen Klini- ken ( $\triangleright$ Abb. 2-6), zum Teil wurde die Augenklinik separat fotografiert ( $\triangleright$ Abb. 7$\mathbf{9}, \mathbf{1 1})$. Ansichtskarten von anderen Gebäudeperspektiven sind nicht bekannt. Auf den Ansichtskarten ab 1930 ( $\triangleright$ Abb. 6 und 11) ist der 1927/28 erbaute, gegenüber dem Hauptgebäude etwas flachere
Anbau nach Westen zu sehen. Ansonsten erfolgten bis zuletzt keine wesentlichen, äußerlich sichtbaren Baumaßnahmen an der Klinik selbst. Lediglich einige Schornsteine wurden nach 1940 abgetragen. Der zwischen 1909 und 1921 angelegte Treppenaufgang (vgl. - Abb. 7 und 9) ist bis heute erhalten.

Aus den Postkarten wird der grundsätzliche Wandel im Krankenhausbau ersichtlich. War es bis in die Weimarer Republik hinein noch üblich, dass jede medizinische Fachdisziplin ihr eigenes Gebäude hatte, so wurden nach dem Zweiten Weltkrieg zunehmend Großbauten populär, die verschiedene Fachdisziplinen unter einem Dach vereinten. Die im deutschen Kaiserreich errichteten „Fach-Kliniken“ zeichneten sich durch eine dezentrale Versorgung, also z. B. eigene Küche und Wäscherei, sowie, auf den Ansichten gut zu sehen, großzügige Grünanlagen in der Umgebung aus [3,5-7]. Während die Krankenhausbauten der "Jetztzeit“ meist außerhalb des Stadtzentrums gelegen sind, bestand seinerzeit noch eine räumliche Nähe zur Universität in der Stadtmitte, die dem „akademischen Selbstverständnis“ sicherlich zuträglich war.

Die Ansichtskarten sind zum Teil vorn, vor allem aber auf der Rückseite beschrieben. Die Karten, die mehrere Kliniken zeigen, wurden i. d. R. von Patienten anderer Kliniken oder auch von Besuchern Tübingens genutzt, während die Postkarten, welche die Augenklinik separat zeigen, zumeist von Augenpatienten oder von Mitarbeitern der Klinik verwendet wurden.

Die letzte Postkarte von der 2. Augenklinik wurde ca. 1980 vom Verlag „Gebrüder Metz" in Tübingen produziert und bis 2015 an der Pforte der Klinik verkauft. Sie konnte aus urheberrechtlichen Gründen nicht publiziert werden. Die Perspektive war nahezu die gleiche wie die von - Abb. 11.

Gustav Schleichs Klinik von 1909 wurde am 20. Oktober 2016 für den Patientenverkehr geschlossen. Das Gebäude wird nach der Renovierung geisteswissenschaftliche Institute der Universität beherbergen, im Tübinger Volksmund aber voraussichtlich „die Augenklinik“ bleiben. 


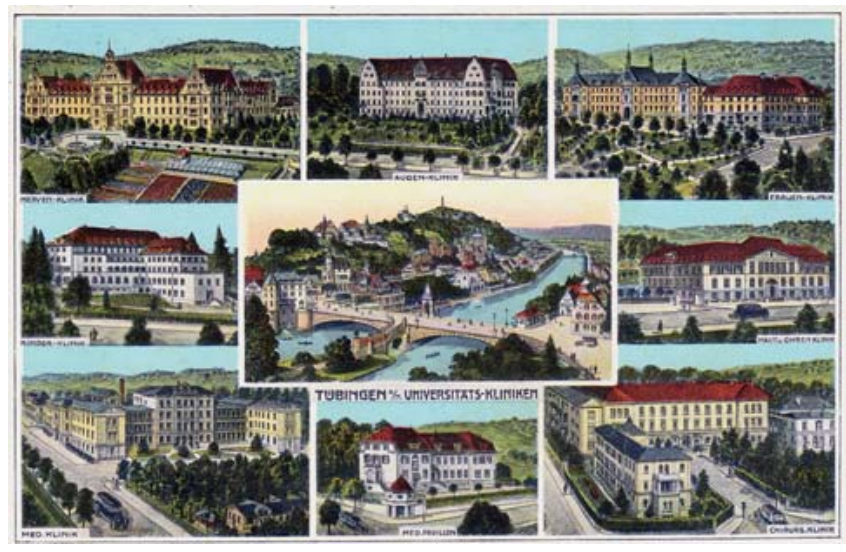

- Abb. 5 Tübinger Kliniken, nunmehr auch mit der Kinderklinik, auf kolorierter Postkarte. Augenklinik erneut oben mittig. Im Zentrum Ansicht von Tübingen mit Neckarbrücke und Österberg. Die Postkarte stellt damit den Bezug von Kliniken und Stadt her. Briefmarke und Poststempel von 1930

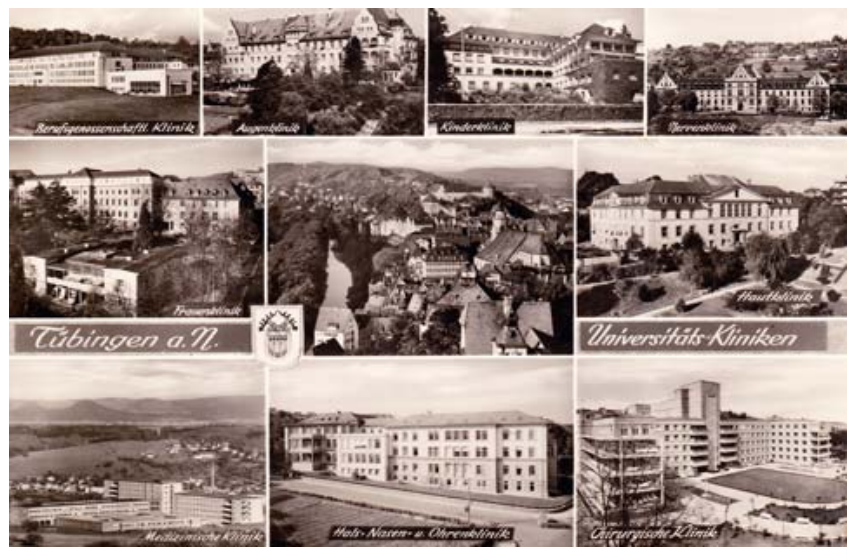

- Abb. 6 Augenklinik oben Mitte links. Mit der berufsgenossenschaftlichen Unfallklinik und der Medizinischen Klinik sind Neubauten hinzugekommen, während alle anderen Kliniken ihr äußerliches Gesicht noch bewahrt haben. Im Zentrum Ansicht des Tübinger Schlossbergs mit Neckar, Stiftskirche und Schloss Hohentübingen. Damit unterstreicht auch diese Postkarte die „Symbiose“ von Kliniken und Stadt. Undatiert, ca. 1965.

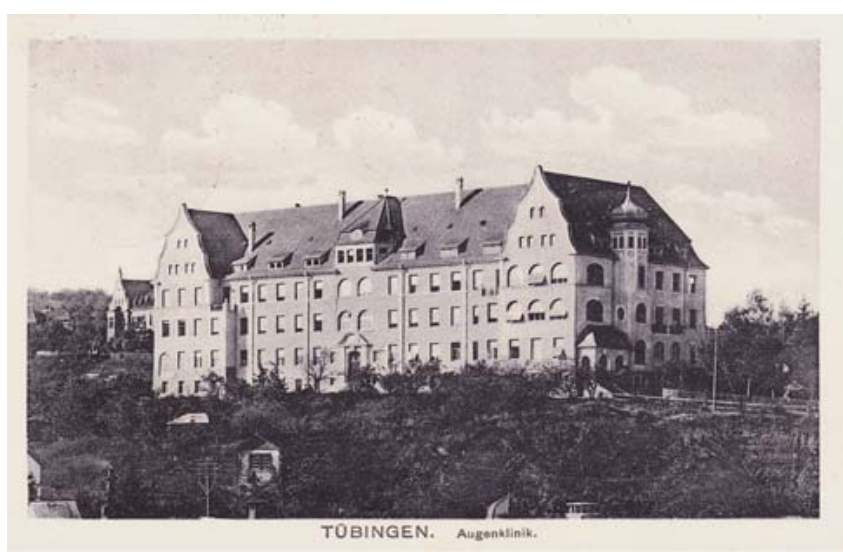

Abb. 8 Weitgehend identische Perspektive wie in $>$ Abb. 7. Datierung und Poststempel vom August 1917. Da der Erste Weltkrieg zu dieser Zeit noch tobte und es sich beim Schreiber um einen Angehörigen oder Patienten des in der Klinik befindlichen Reservelazaretts handelte, wurde die Postkarte als „Feldpost“ verschickt.
- Abb. 7 Postkarte mit der neuen, 2. Augenklinik als einzigem Gebäude. Der Schreiber berichtete von seinem Aufenthalt in der Klinik. Mit dem Kreuz wurde sehr wahrscheinlich das Krankenzimmer markiert, in dem der Patient lag. Poststempel vom 21. Dezember 1909. Es dürfte sich daher sehr wahrscheinlich um die erste Postkarte der Klinik, die am 1. Januar des gleichen Jahres in Betrieb genommen worden war, handeln.
Ob es der Neubau auf dem Schnarrenberg jemals auf eine Ansichtskarte schaffen wird, bleibt abzuwarten. Das Internet, die heute nur kurze stationäre Verweildauer und der Umstand, dass Krankenhausaufenthalte in der Universitätsstadt Tübingen ganz anders als vor Jahrzehnten heute keine wirklichen, berichtenswerten „Highlights“ im Leben mehr sind, sorgen dafür, dass auf Ansichtskarten verfasste „freundlichste Grüße aus der Augenklinik“ im Wesentlichen nur noch nette, historische Reminiszenzen sind.
Interessenkonflikt

Die Autoren geben an, dass kein Interessenkonflikt besteht.
Autoren

Jens Martin Rohrbach ${ }^{1}$, Karl Ulrich Bartz-Schmidt ${ }^{2}$, Conka llieva Tekeva-Rohrbach ${ }^{3}$, Martin Alexander Leitritz ${ }^{2}$

1 Department für Augenheilkunde, Forschungsbereich Geschichte der Augenheilkunde/Ophthalmopathologisches Labor, Eberhard-KarlsUniversität Tübingen

2 Department für Augenheilkunde, UniversitätsAugenklinik, Tübingen

3 Augenpraxis, Augenzentrum Tuttlingen, Hechingen 


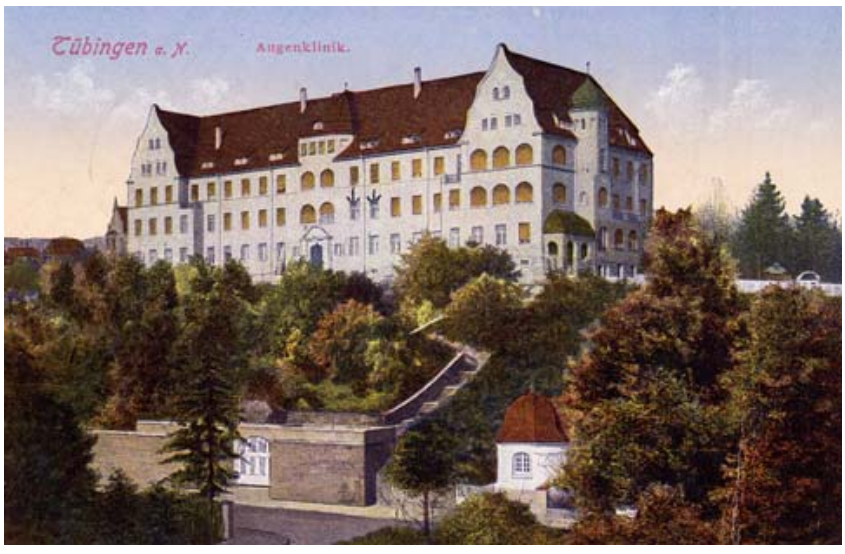

- Abb. 9 Kolorierte Postkarte mit ähnlicher Ansicht wie in \ Abb. 7 und 8. Poststempel vom 21. Mai 1921.

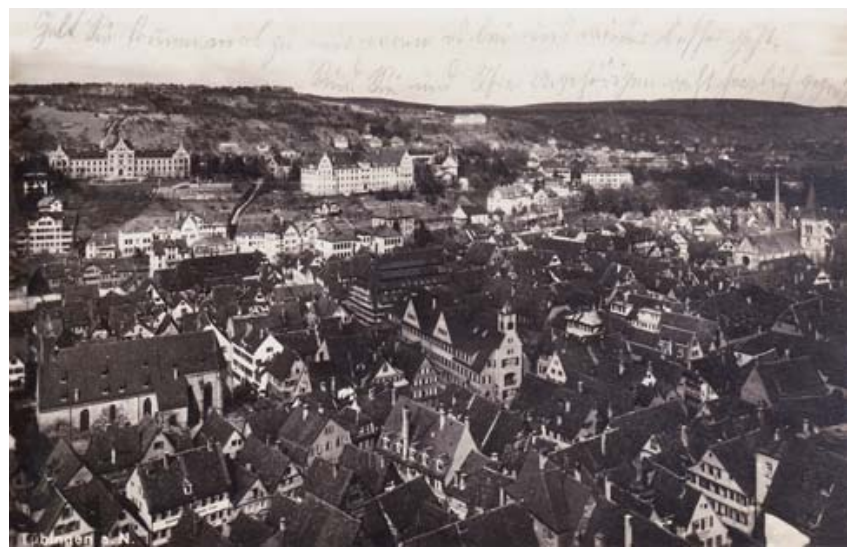

- Abb. 10 Postkarte mit Blick vom Schlossberg auf die Tübinger Altstadt und die Augenklinik leicht oberhalb des Zentrums. Die Augenklinik ist hier „eher zufällig“ auf dem Bild. Man erkennt aber gut, dass sie für das Stadtbild prägend ist. Datierung und Poststempel vom November 1930. Da der Anbau nach Westen noch nicht zu sehen ist, muss die Karte aber aus der Zeit vor 1928 stammen.

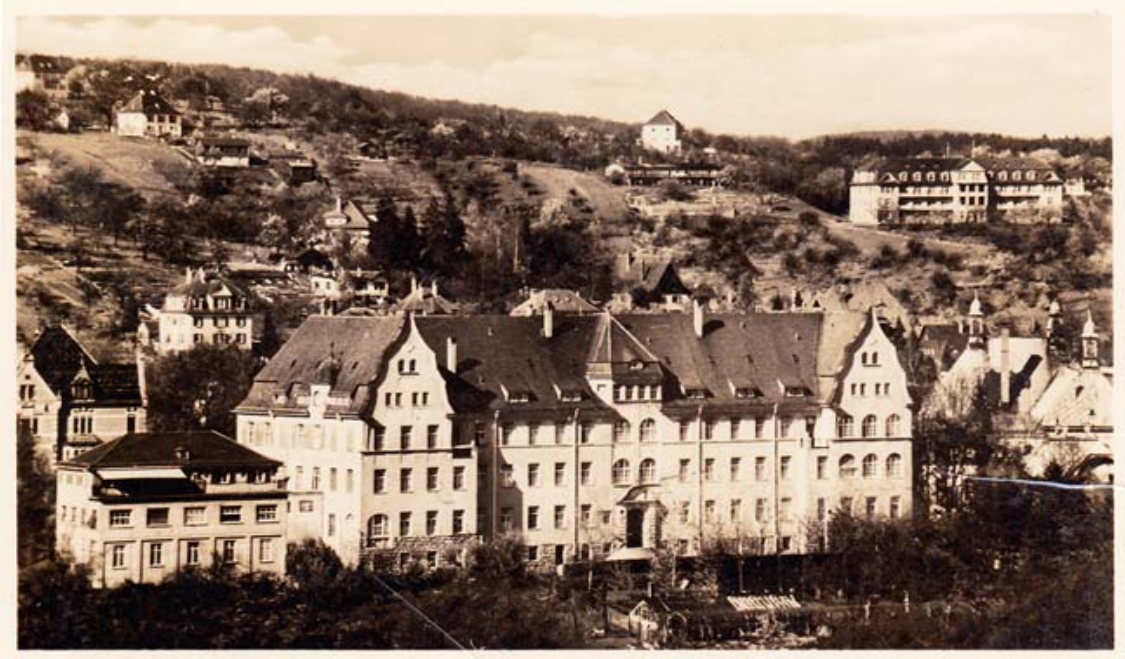

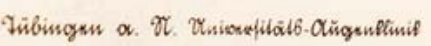

- Abb. 11 Zweite Universitäts-Augenklinik Tübingen, nunmehr mit dem 1927/28 erfolgten Anbau nach Westen. „Tante Klara“ mit Absender „Univers. Augenklinik Saal F“ führte auf der Karte aus: „Muss etwa 3-4 Wochen hier bleiben. Werde tüchtig in Kur genommen. Man kann hier allerhand sehen. Ich habe hier noch viele Leidensgenossen“. In der Tat betrug die stationäre Verweildauer in allen Augenkliniken damals - in der Zeit vor Operationsmikroskop, brauchbarem Nahtmaterial und wirksamen Pharmaka - sehr oft viele Wochen. Datierung auf den Februar 1940.

\section{Literatur}

[1] Nagel A. Die Augen-Klinik. In: Universität Tübingen: Die unter der Regierung seiner Majestät des Königs Karl an der Universität Tübingen errichteten und erweiterten Institute der naturwissenschaftlichen und medizinischen Fakultät. Tübingen: Eigenverlag der Universität; 1889
[2] Mackensen G. 1904-1964. Fortschritte der Augenheilkunde im Spiegel der Württembergischen Augenärztlichen Vereinigung. Tübingen: Eigenverlag der Universität; 1964

[3] Hunder AE, Rohrbach JM, Bartz-Schmidt KU. 100 Jahre Universitäts-Augenklinik Tübingen. Wandel und Visionen 1909-2009. Stuttgart: Schattauer; 2010

[4] Fichtner G. Des Nagels Kopf - die Anfänge der Universitäts-Augenklinik Tübingen. Son- derband „100 Jahre Augenheilkunde in Tübingen". Attempto (Zeitschrift der Eberhard-Karls-Universität Tübingen) 1975; 55 / 56: 50-61

[5] Schleich G. Die neue Universitäts-Augenklinik in Tübingen. Medicinisches Correspondenz-Blatt des Württembergischen ärztlichen Landesvereins 1910; 80: 197-203

[6] Schleich G. Die neue Universitäts-Augenklinik in Tübingen. Klin Monatsbl Augenheilkd 1909; 47: 610-617

[7] Rohrbach JM, Szurman P, Bartz-Schmidt KU. 100 Jahre Universitäts-Augenklinik Tübingen. Klin Monatsbl Augenheilkd 2008; 225: 975-982

\section{Bibliografie}

DOI https://doi.org/10.1055/s-0043-106296 Online-publiziert 2.6.2017 | Klin Monatsbl Augenheilkd 2019; 236: 195-198 @ Georg Thieme Verlag KG Stuttgart · New York | ISSN 0023-2165

\section{Korrespondenzadresse}

Prof. Dr. Jens Martin Rohrbach Department für Augenheilkunde, Forschungsbereich Geschichte der Augenheilkunde/Ophthalmopathologisches Labor Eberhard-Karls-Universität Tübingen Elfriede-Aulhorn-Straße 7

72076 Tübingen

Tel.: + 49/(0) 7071/2984761

Fax: $+49 /(0) 7071 / 294762$

Martin.Rohrbach@med.uni-tuebingen.de

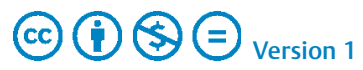

